\title{
Connecting Early VLBI to SKA capabilities
}

A. van Ardenne ${ }^{1}$

ASTRON, Chalmers University of Technology

Postbox 2, 790 AA, The Netherlands

E-mail: ardenne@astron.nl

Several technical developments of early VLBI are relevant and now common for pathfinder and other initiatives toward the SKA. While no surprise when wearing a general "progress" hat, direct connections have so far not been stipulated or pointed out explicitly.

In this contribution, I will reflect on some of these aspects of early VLBI developments which lead to relevant developments for the SKA and to which as seen from Dutch VLBI and beyond, Richard played a crucial facilitating role as well as a key role to the SKA endeavours. As I was connected with VLBI instrumentation developments in the Netherlands from the beginning, no doubt a personal view may bias some of its perceived importance. Nonetheless there can be no misunderstanding regarding Richards crucial and energetic role directly and indirectly in connecting VLBI with space to achieve even higher resolution imaging, higher sensitivity observing through so called tied array telescopes with the Westerbork imaging synthesis array, to exploring wider frequency coverage with these telescopes and of course in setting up the organization and managerial frameworks toward VLBI and JIVE in Europe.

Here I will go into some detail regarding relevant technical aspects of VLBI for the SKA with emphasis on aperture (phased) arrays leaving the "shaping up" frameworks for others to address.

Resolving the Sky - Radio Interferometry: Past, Present and Future

Manchester, UK

April 17-20, 2012

\footnotetext{
$1 \quad$ Speaker
} 


\section{Introduction}

In the mid-seventies the first VLBI observation in the Netherlands was planned for by George Miley and Boudewijn Baud with involvement of Harm Habing, at the time all working at the Leiden Observatory. This became a practical possibility by the interest and experience of Richard Schilizzi as newly arrived postdoc from Caltech in 1975 working together with the author from NFRA's (now ASTRON's) technical labs.

With NRAO's help by providing a MKII IF-converter and formatter and an AMPEX 2" taperecorder system and a Rubidium clock plus television time synchronization from the Kootwijk (in Nl.) based geodesy department of Technical University Delft, an $18 \mathrm{~cm}$ observation was scheduled using the $25 \mathrm{~m}$ Dwingeloo telescope and the cryogenically cooled receiver. See Figure 1 below. Other participating VLBI stations were using Onsala's 25m and MPI's Effelsberg 100m telescope.

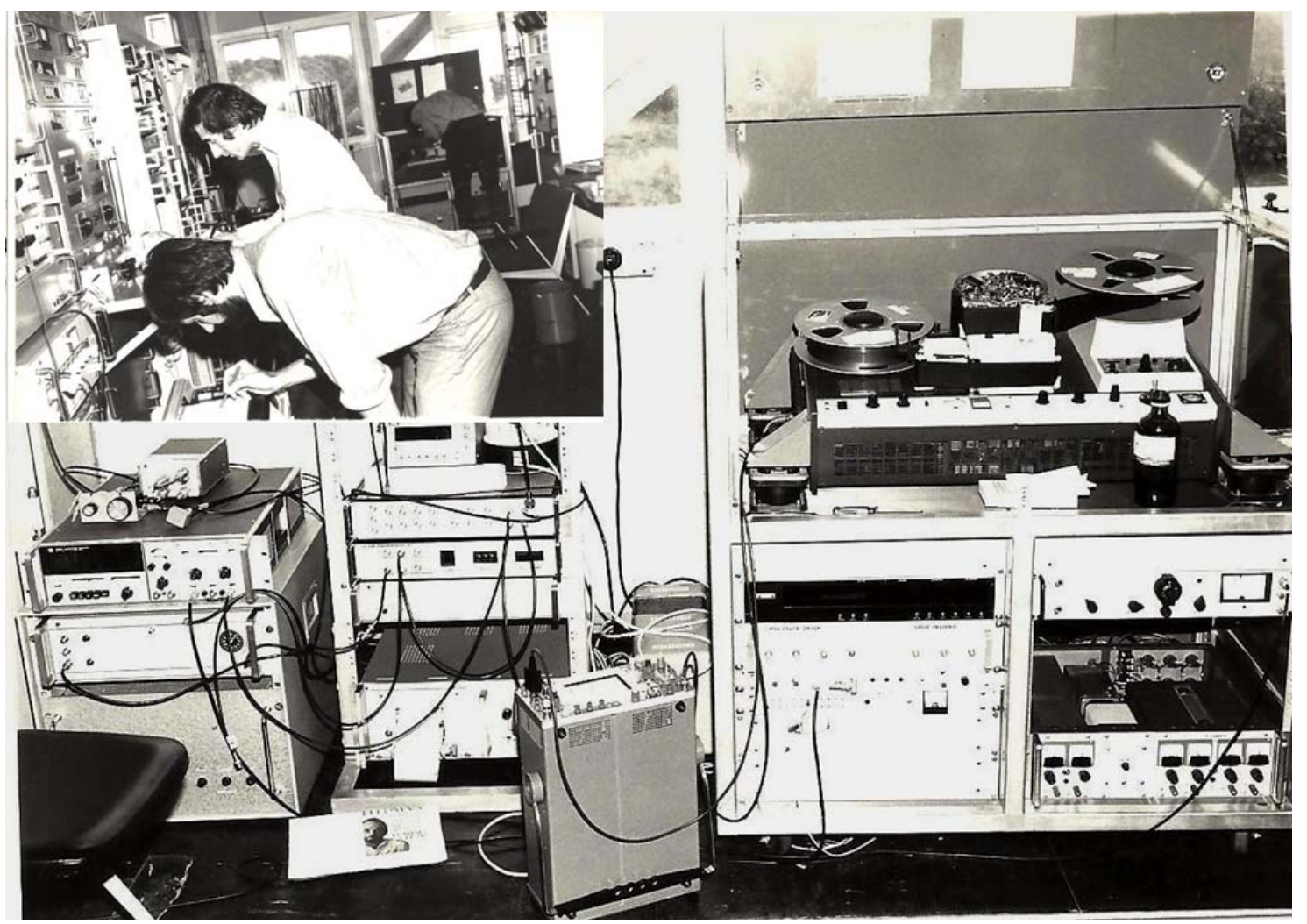

Figure 1: The first VLBI observation (then called "experiment") at $18 \mathrm{~cm}$ in the Netherlands in 1976 inside the receiver cabin of the Dwingeloo Alt-Az telescope using borrowed VLBI equipment (see text) from NRAO and the Technical University Delft. Richard Schilizzi is depicted in the insert together with initiators George Miley and Boudewijn Baud in the background.

Data was processed using NRAO's 2-station VLBI correlator in Charlottesville resulting in a 3C236 A\&A paper [1] some time later. Thanks largely to Richards continuing efforts, this successful event marked the beginning of intense VLBI involvement from the Netherlands 
contributing to the establishment of the European VLBI Network ("EVN") (www.evlbi.org ) and JIVE (www.JIVE.org) some 15 years later reaching a global scale of activities.

VLBI may be seen as an observing technique built onto and with a few exceptions in particular for geodesy, using existing telescopes thereby exploring major new fields to serve its purpose. For example. real-time high data techniques though fiber (keeping photonics technologies within the horizon of radio applications), data storage techniques, time/phase dissemination, and (deep)space relevant applications. In the SKA, given its requirements of long baselines/high resolution observing in real-time, some of these techniques must be intrinsic to its design. See Table1.

\begin{tabular}{|c|c|}
\hline Early VLBI for high resolution observing & Toward SKA \\
\hline - $\quad$ Limited number of baselines & - $\quad$ Many baselines; excellent uv-coverage \\
\hline - $\quad$ Limited Bandwidth 1MHz & - $\quad$ High bandwidth $\sim>\mathrm{GHz}$ \\
\hline - $\quad$ 1- bit Taperecorders & - $\quad$ Real-time through fiber networks \\
\hline - $\quad$ Post-observing processing & - $\quad$ Real-time processing and observing \\
\hline $\begin{array}{l}\text { - Time sync. through Long wave receivers } \\
\text { (LORAN, DCF77) \& TV time } \\
\text { dissemination }\end{array}$ & $\begin{array}{l}\text { - Time diss. through GNSS, other? } \\
\text { - High stability phase coherency through } \\
\text { fiber? }\end{array}$ \\
\hline $\begin{array}{ll}\text { - } & \text { Single telescope as station } \\
\text { - } & \text { Heterogeneous telescopes }\end{array}$ & $\begin{array}{l}\text { - Complex mixed-mode observing e.g. } \\
\text { including transients and imaging } \\
\text { - Homogeneous in SKA core, possibly } \\
\text { heterogeneous for longest BL's and } \\
\text { highest resolution? }\end{array}$ \\
\hline $\begin{array}{l}\text { - Limited polarimetry, limited Dynamic } \\
\text { Range }\end{array}$ & - $\quad$ Highest DR polarimetric imaging \\
\hline
\end{tabular}

Table 1: Comparing early VLBI capabilities with SKA requirements and expectations. For the SKA aspects normally part of VLBI are now to be designed in drawing from experiences and techniques explored in VLBI.

Work has been going on to explore concepts leading to the most science capable SKA for which it could be said that paradigm shifts operate on a combination of performance dimensions like high Point-source Sensitivity, Ultra-deep and Polarimetric Imaging, Observational Flexibility including time-variable observing, Extensive field of view allowing for simultaneous Multi- Sky pixel observing. As an example, Figure 2 depicts an exploration of several observing modes by J-P. Macquart [2] desirable to observe the transient sky.

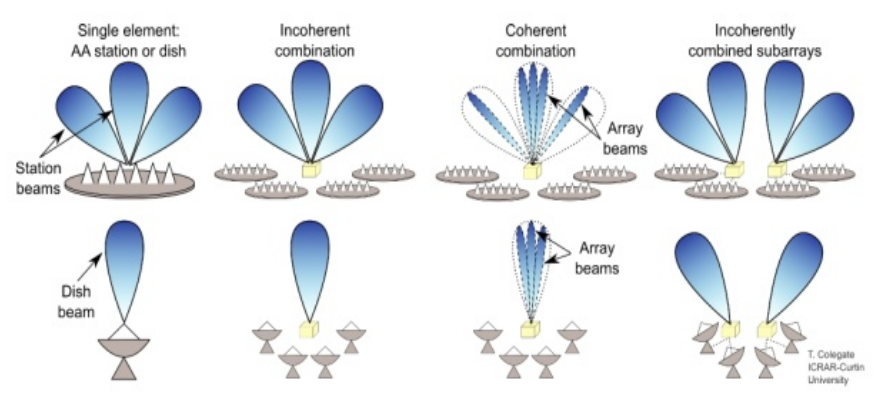

Figure 2: Exploration of several observing modes. Courtesy J-P Macquart[2] 
Such efforts do not come for free including a relatively long lead-up time toward realizing this most flexible SKA instrument. It is therefore relevant to focus on some important aspects for which the groundwork was laid so much earlier and which support the technical progress toward the SKA perpetuated through Richards driving force.

\section{Relevant and important aspects}

In this section some aspects are explored as examples from early VLBI bearing immediate relevance for the SKA. These of course constitute a choice partly induced by their (almost forgotten?) relevance and/or because of their visionary implications. It is by no means an exhaustive or detailed treatise but rather explore the outcomes and possible implications for the SKA.

\subsection{Digitization, Correlation and loss of sensitivity}

\subsubsection{The early case of 1 bit correlation}

In the simplest case of calibrating the correlated flux between received signals from two, say, parabolic telescopes, on a VLBI baseline, the following general equation holds:

$S_{c}=2 . b$. .k. . $\left(\frac{T s 1}{\eta 1 \mathrm{~A} 1} \cdot \frac{\mathrm{T} s z}{\eta 2 \mathrm{~A} 2}\right)^{1 / 2}$

$$
\begin{aligned}
& T_{\mathrm{si}}=\text { system temperature, } \mathrm{i}=1,2 \\
& \eta_{\mathrm{i}}=\text { aperture efficiency, } \mathrm{i}=1,2 \\
& \mathrm{~A}_{\mathrm{i}}=\text { physical telescope area, } \mathrm{i}=1,2 \\
& \mathrm{Q}=\text { measured correlation coefficient as } \\
& \text { derived from the digital correlator } \\
& \mathrm{k}=\text { Bolzmann factor } \\
& \mathrm{b}=\text { "fudge" factor to make it all fit }
\end{aligned}
$$
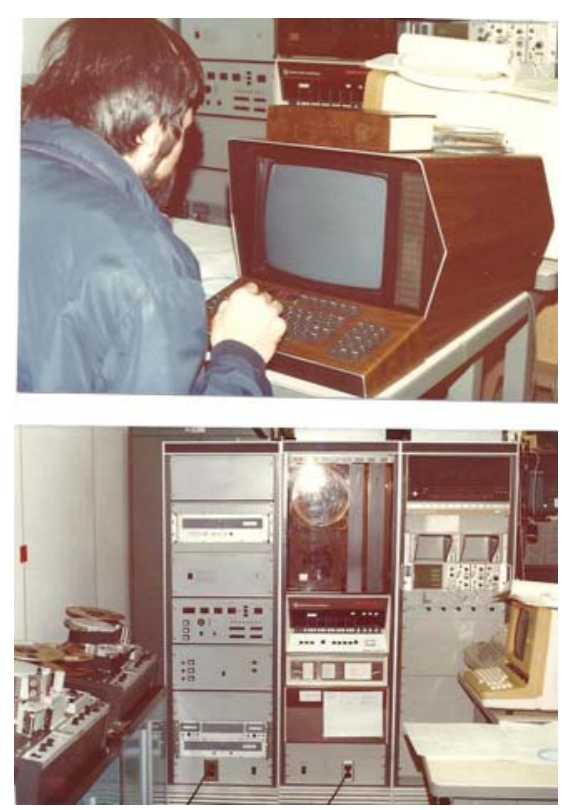

Figure 3: Correlating the first VLBI observation at the two station NRAO correlator in 1977; the "fudge" factor derived from an unresolved point-source makes it all fit.

Such equation was also used by Richard in Figure 2 to fit the correlated fluxdensities to the newly observed source data for which the introduction of the so-called fudgefactor was required. This factor effectively absorbed all factors including the digitization loss by for 
example using a e.g. single bit (2-level) correlator in which case is it somewhere between 2.12.5. This was considered acceptable considering the benefit of high resolution as key observational result.

Although not clear from equation 1, this fudge factor effectively constitutes a sensitivity loss and therefore should be as close as possible to 1 for which case the "measured" correlation equals the "true" correlation.

For a tied array in which quantized signals are properly added in phase through which information and therefore an "excess" sensitivity loss is incurred before the summed signal is quantified as one leg input of the single baseline (to keep the simplest case) correlator. The matter then becomes more complicated and has interesting ramifications.

\subsubsection{Correlation and loss of sensitivity; the case of Westerbork as tied array, VLBI and Pulsar}

It is possible to add all telescopes signals in-phase thereby achieving a much increased sensitivity for VLBI. In case all signals are added such as for the Westerbork array of telescopes of $14 \times 25 \mathrm{~m}$ dishes, a tied array is formed with a maximum equivalent diameter of $93 \mathrm{~m}$ (see Figure 4), approximating a similar sensitivity as the largest movable telescopes like the $100 \mathrm{~m}$ Effelsberg telescope (and later the GBT). Such a system was first implemented in Westerbork fully analog end-seventies and subsequently as a digital system using the already installed "standard" A/D converters before adding digitally. In the process information is lost and an

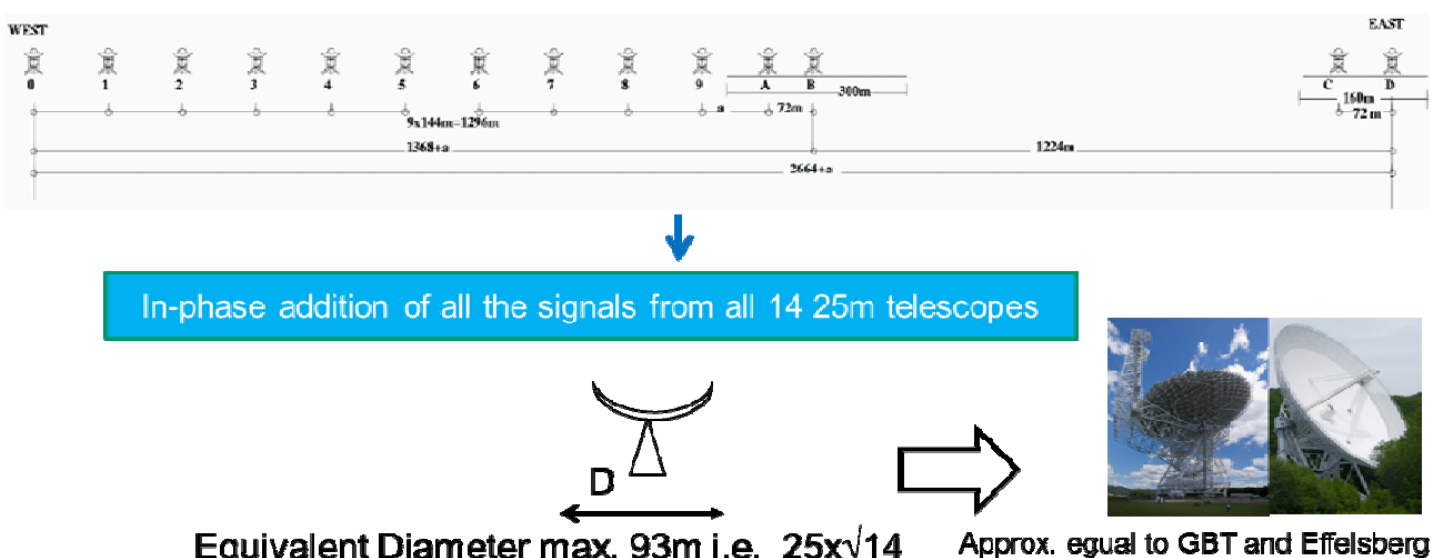

Figure 4: Depicting the Westerbork array as one single telescope achieving a maximum equivalent receiving diameter roughly comparable with the Effelsberg 100m and later, the GBT telescope.

"excess" degradation in the tied array arm is incurred beyond the "common" loss expressed in the fudge factor " $b$ " mentioned earlier. This issue was recognized early on by the author and its consequences were first investigated and written up in various NFRA notes over 1978-1982 [e.g.3] and subsequently detailed in a much later 2001 paper[4]. The arrays phase centre for VLBI coincides with the delay-and fringe stopping centre and for practical reasons (i.e only positive natural delay rates) is chosen to be east from the array. 
As a corollary of these tied array VLBI oriented developments induced by Richard, it became also clear that in parallel to doing aperture synthesis required for calibrating the tied array, it was possible to use the tied array signals for e.g. pulsar observations [5] and in fact through Richard Strom and Hans van Someren Greve, actually doing so in parallel to aperture synthesis[6]. Ultimately, this early work stimulated the developments into making Westerbork a flexible pulsar observing machine starting through initial PUMA work in the nineties by Loudie Voûte and others[7] and contributing to a vital pulsar community in the Netherlands.

\subsubsection{From tied array to the SKA, lessons learned}

In the 2001 paper[4] on tied arrays and excess digital degradation, Figure 5 below was used to describe the general situation as seen from the signal paths. Proper recognition was given to the total power from the tied array and the correlation on VLBI baselines. The paper introduced the concepts of effective Antenna area and the effective system noise important to calibrate the array.

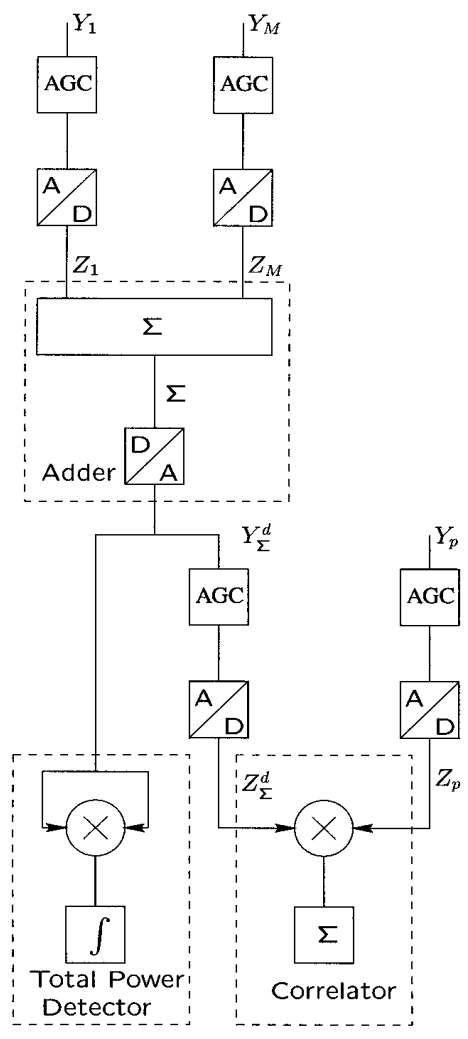

Figure 5: General description of the Westerbork tied array with digitization before further processing in this case correlating with another (VLBI) telescope. The latter could of course also be the result of a tied arraying arrangement. Source: [4]

It concluded that the maximum sensitivity was reached with equal antennas/receivers AND analog (rather than digital) addition. In the case of Aperture Arrays for the SKA [e.g.8] Figure 5 could be seen as a simplest case for consecutive stages of digitization. For example in Aperture Arrays analog-beamforming is considered for reasons of e.g. cost and required processing while here to this the argument for lower loss is added to that. 
More quantitatively, and as example from [4],10\% loss with 14 telescopes is incurred with 4 bit A/D conversion which also was the limit case for the analysis. At this stage one obvious conclusion is therefore that more bits are needed to minimize loss and secondly that more receivers are needed before a next stage digital processing. More specifically (and also for the SKA), the "loss" is limited to a few per cent for more than 5 bit A/D with the number of combined array elements should be larger than, say, 15-16.

For the SKA the number of elements is large, say, tens to hundreds while 8-12 bit quantization schemes have been considered thus far. Apart from the possible need to look into this more thoroughly e.g the effect of averaging, other remaining issue are (i) how far this loss manifests itself as sidelobes[e.g.9] and/or (ii) calibration consequences also for tied SKA-dish designs. Note also that LOFAR as aperture array is extensively looking into and exploring the use of (multiple) tied array beams[10] for pulsar research work and it can only be expected this capability holds for the SKA as well!

\subsection{Connecting Clockstability with VLBI at highest resolution}

\subsubsection{Coherency loss, VLBI and tied arrays}

In an important paper Allan Rogers and Jim Moran investigated the limits of phase coherence in VLBI and clockstability performance resulting from the independent station clocks operating as auxiliary, local oscillator generators[11]. This allowed to make estimates of coherent integration times on VLBI baselines at any observing frequency. For example, for $\mathrm{H}-$ masers this loss at $10 \mathrm{GHz}$ is $5 \%$ or less and better at lower frequency, See Figure 6 below.

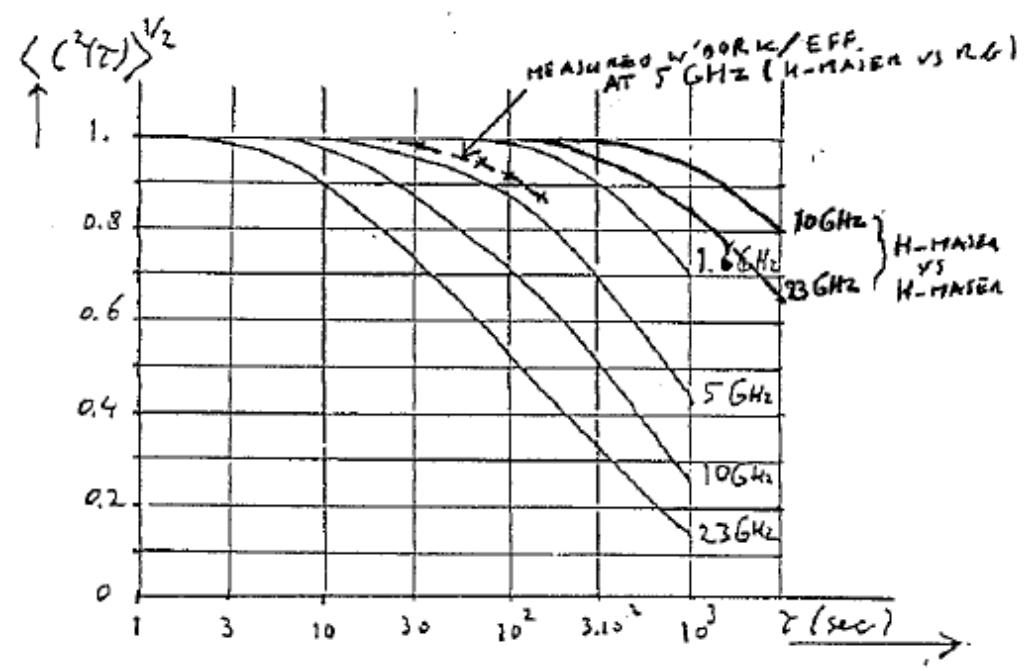

Figure 6: VLBI coherence for a $\mathrm{H}$-maser/Rb standard and a $\mathrm{H}-\mathrm{maser} / \mathrm{H}-$ maser combination as a function of integration time with the observing frequency as parameter. The curves are based on the analysis in[11,12]. It also shows some measured data from Westerbork with Effelsberg as second station.

This of course, was timewise commensurate with developments toward the tied array developments in Westerbork in response to Richards call for higher sensitivity VLBI observations. This ambition implied investigating the local oscillator and in 
general the phase stability of the Westerbork system designed with local interferometry in mind for which "only"differential effects are important and not as a well designed VLBI station in which the "absolute" stability matters. For this to access, the Rogers/Moran paper [11] was important as well as for other matters to be discussed below. A full phase stability assessment was done[12] when turning Westerbork into a MKIII compatible system used in conjunction with a new wideband so called DCB correlator of which John O'Sullivan was the originator. The result was positive i.e. the system overall turned out to have approximately equal stability performance as from the H-maser only used in the (VLBI) tied array.

As a corrolary to this kind of stability analysis[12] one may think of consequences of "stacking" HI data from independent data, now commonly done. Without making any judgement on its exciting results, this procedure means weighting the data with an (unknown) transfer function (probably not important) and being integrated intensity data, results in a fourth power vs. system temperature averaging law which does not seem optimal.

\subsubsection{Highest resolution VLBI; QUASAT and Phase transfer using satellites}

Without aiming to be fully correct nor complete, this section is about the next step Richard put his full energy into and indeed involving many others.

Early eighties concepts were proposed to perform highest resolution VLBI observing through the use of orbiting VLBI station inially notably from the US and Canada[e.g.13] $]^{2}$ The concept evolution involved many astromers and engineers from the US and Europe and both space agencies NASA and ESA studying the Quasar Satellite ("QUASAT") concept. After technical studies [14], this resulted in a proposed Phase A mission in October 1988 of which the cover is shown in Figure 7 depicting the mission concept outline as described in [15 and references].

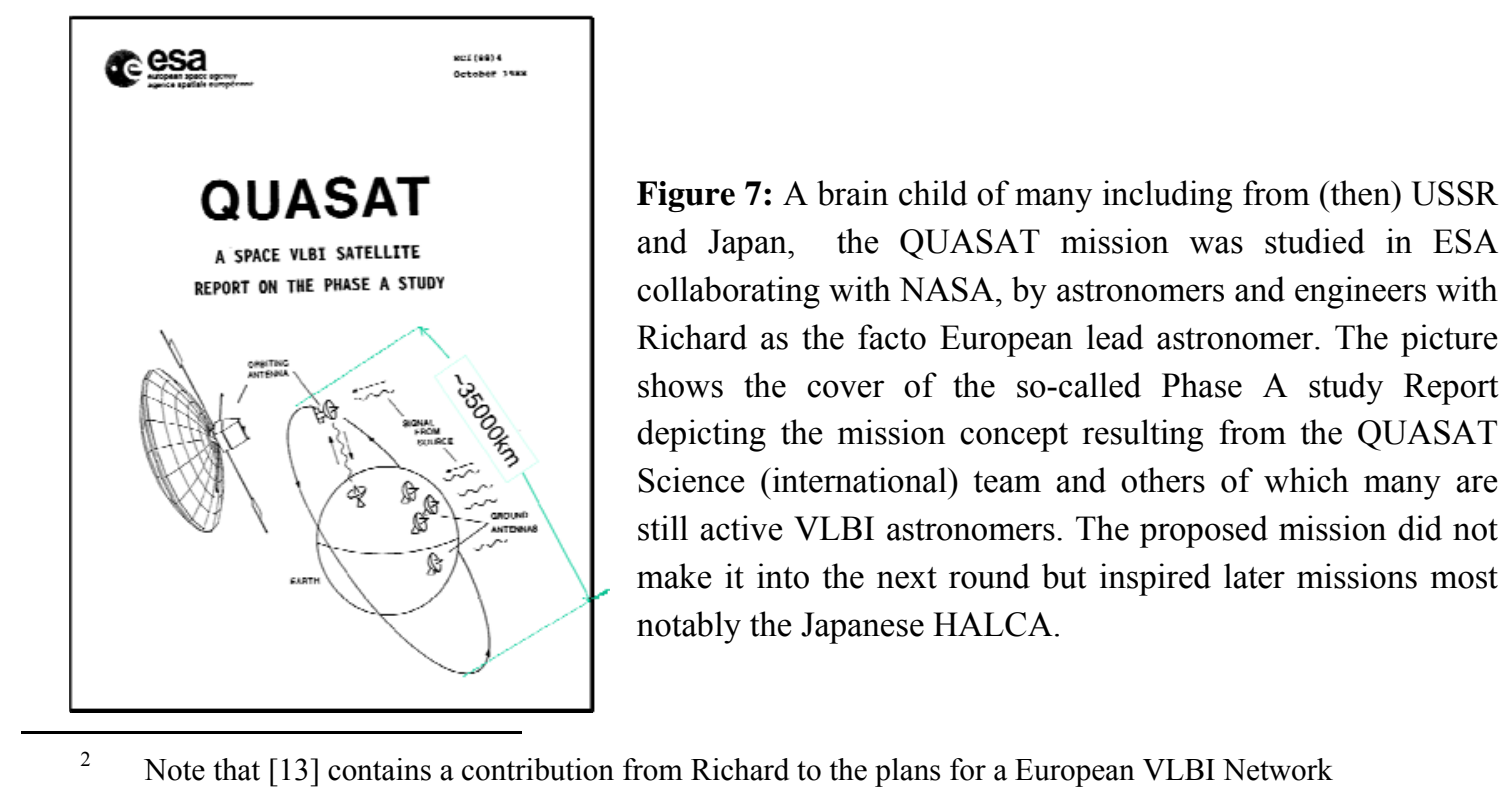


Although the proposed mission did not make it into ESA further main mission planning favouring other exciting proposals notably the CASSINI Saturn mission launched in $1997^{3}$, QUASAT did inspire efforts for succesful later missions to come. While planning for the (now) succesfully launched Russian space project RADIOASTRON for highest resolution VLBI continued, this notably was the case for the Japanese lead VSOP later officially named "HALCA" now made inoperative.

\subsubsection{Highest resolution VLBI; Phase transfer using satellites}

The plans and ideas for a space-based VLBI station and the cost of stable H-maser clocks stimulated earlier ideas[16] to investigate coherent phase transfer through communication satellites in the eighties. Activities were set up notably in Canada and somewhat later in the Netherlands and again later in Australia all being succesful in proven the principle and some showing better performance than the H-maser reference.

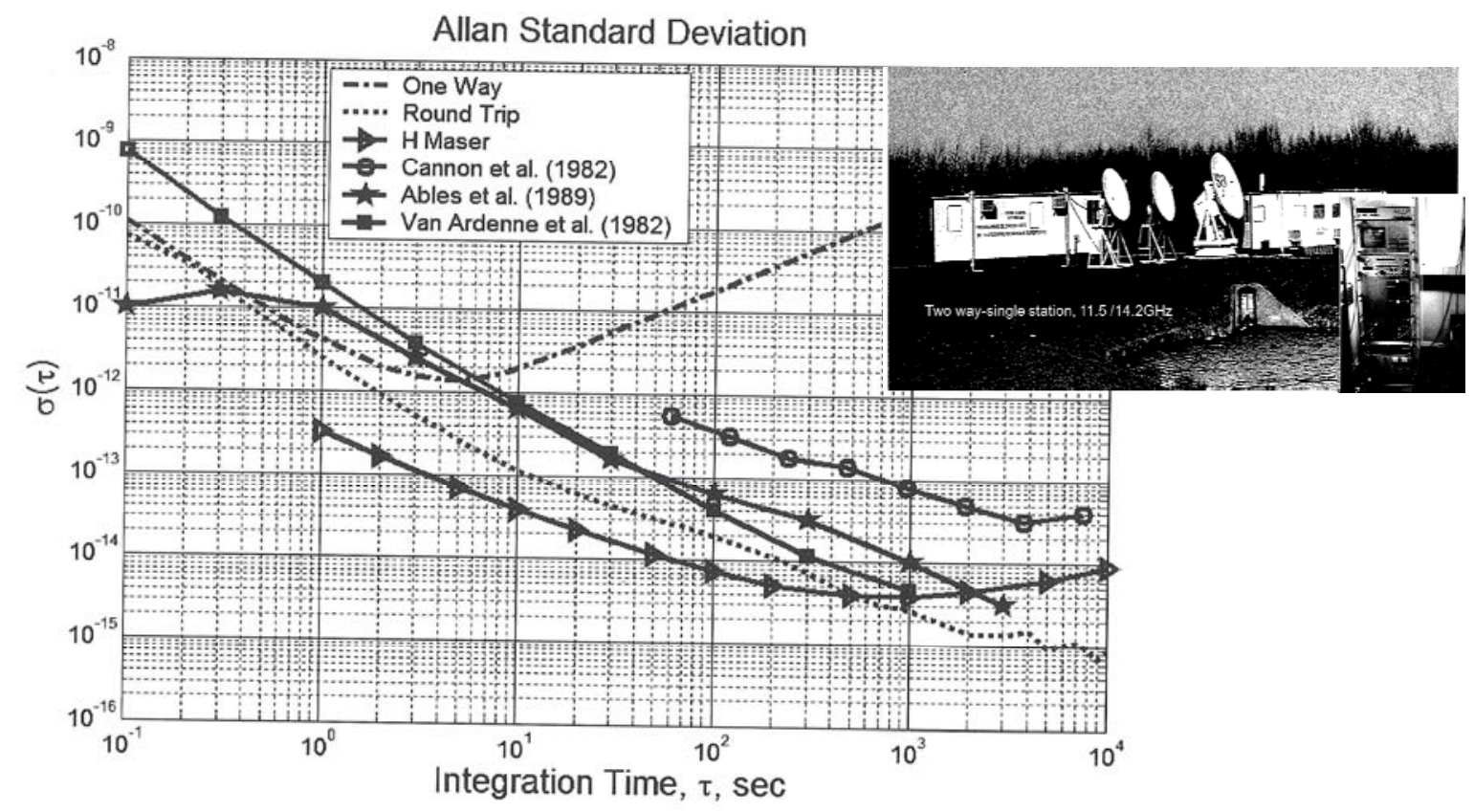

Figure 8: From Bardin et.al. [17]: A comparison of successive phase transfer results using various communication satellites in order to look into possibilities to use a single VLBI clock. Some showing better performance than the H-maser after a thousand seconds. The most recent result from [17] is shown as dotted line. As is commonly done the performance measure is expressed as the socalled Alan variance versus integration time. The insert shows the cabin and equipment used in performing the 1981 OTS-2 experiment in the Netherlands on the ESTEC premises.

In the context of investigating technologies for the SKA, a similar round-trip experiment was done more recently using the Telstar-5 satellite showing that indeed performance of the best available clock for VLBI i.e. the H-maser could be improved on timescales for higher than

3 And to which also VLBI techniques were applied in the context of successful science recovery of the Titan probe 
about 1000 second coherent integration times. See Figure 8 for a comparison of performance results from various experiments[17 and references].

It remains to be seen as a result of the present SKA system design efforts if the concept of satellite based phase transfer is indeed attractive for the long baselines planned for the SKA. Alternatively, on-going SKA efforts aim to investigate the potential of clock transfer using technologies common to high performance, high data fiber optics communications developments.

\subsection{Complex receivers and feed systems for SKA?; another potential spin-off from QUASAT}

This section extrapolates somewhat speculatively to potential SKA applications of developments at the fringe of feed/receiver developments starting in the context of QUASAT technologies and the need to update the Westerbrok receiver system.

For Quasat science it was decided to operate in four frequency bands around 1.35, 6, 18 and $92 \mathrm{~cm}$ wavelenghts from around $22 \mathrm{GHz}$ down to between $0.324-328 \mathrm{GHz}$. Based on technical and mission considerations it was decided to combine systems and explore the use of a triple coaxial feed previously done in Westerbork in a dual feed $6 / 50 \mathrm{~cm}$ arrangement in the seventies.

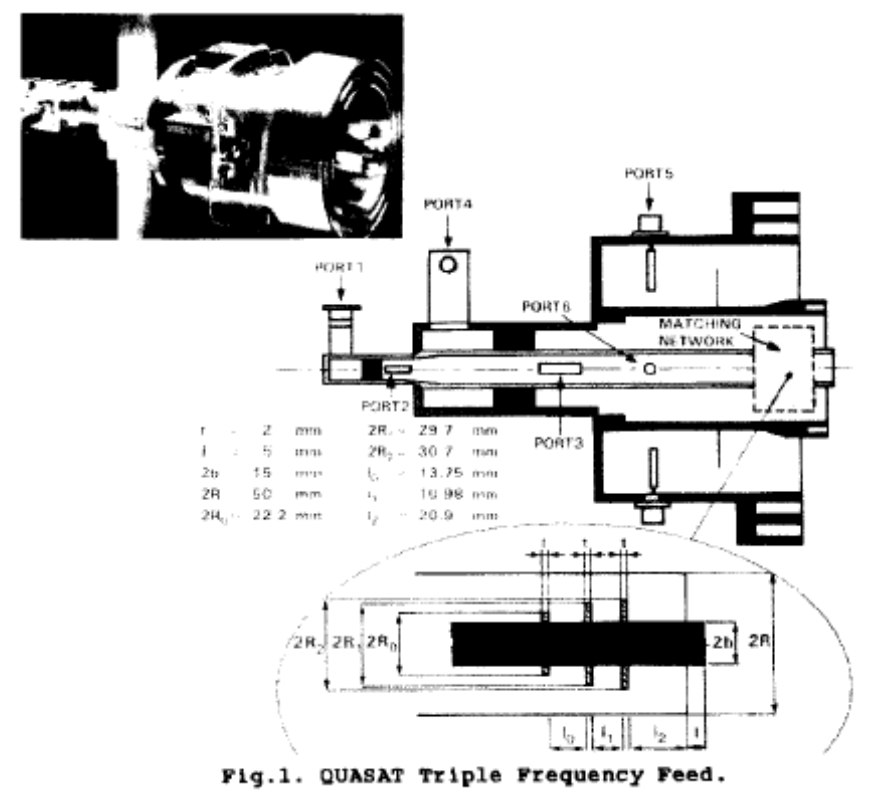

Figure 9: The dual polarized triple frequency band feed system for the Quasat antenna. The feed showed excellent performance and operated around 1.35, 6 and $18 \mathrm{~cm}$ wavelength.

Results were excellent [18] and a picture of the design is shown in Figure 9. Around the same time, it was decided to replace the multitude of receivers in the Westerbork array to reduce the operational load and maintenance, to increase observing 
bandwidths and to increase observing capabilities by accommodating a $\mathrm{S} / \mathrm{X}$ receiving capability required for e.g. geodetic VLBI. This resulted in the so-called multifrequency frontend described in e.g. [19] and shown in Figure 10. It operates in 9 bands from 0.25 to $8.6 \mathrm{GHz}$ with the four bands that require highest sensitivity i.e. $\mathrm{S} / \mathrm{X}, 6 \mathrm{~cm}$ and $18 / 21$ $\mathrm{cm}$ all cryogenically cooled using a carefully designed single cryogenic system with single refrigerator. The $\mathrm{S} / \mathrm{X}$ feed is also combined with the $92 \mathrm{~cm}$ feed as uncooled frontend as a triple coaxial configuration similar to but not the same as in the Quasat feed arrangement, and the cooled $6 \mathrm{~cm}$ is coaxially combined with the $50 \mathrm{~cm}$ feed.

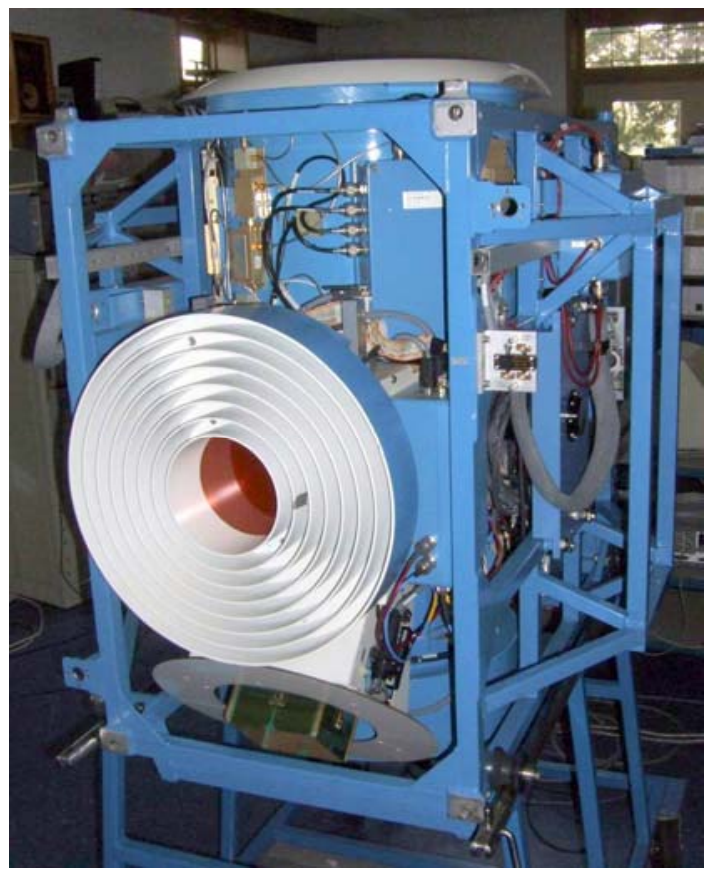

Figure 10: The 9 band Multifrequency frontend used in the Westerbork array. S/X, 6 and 18/21 cm bands are cryogenically cooled in a single cryogenic system. Rapid changes in observing bands are achieved by combining feed systems and by feed rotating mechanism using a single axis turret system. The triple $92 / 13 / 3.6 \mathrm{~cm}$ feed system is looking upward and the dual $6 / 50 \mathrm{~cm}$ feed is looking face-on.

Rapid changes in observing bands are achieved by combining feed systems and by a feed rotating mechanism using a turret system positioning the chosen receiving bands in the prime focus of the $0.36 \mathrm{f} / \mathrm{D}$ system. In this way, a highly flexible and reliable low noise receiver resulted with minimum power consumption and maintenance thanks to the use of "only" 14 receivers and cryogenic systems covering all frequencies!

The system is to a large extent planned to be phased out when accommodating the APERTIF Phased Array frontends in Westerbork in the near future. However, the positive experience with turret approach in combination with the single cryogenic system seems nonetheless a candidate design concept for SKA dishes with a need for multi-feed/multi-band foci. 


\section{Conclusion}

Various aspects of VLBI induced by Richards involvement over many years have been selected and shown to be applicable and useful to the present SKA developments. The work illustrated in the first two sections based on early VLBI experience resulted in essentially a full understanding of coherent phased arrays relevant for present SKA activities. To this, new and other experiences from LOFAR and other arrays like the MWA will of course add to the knowledge base in particular for the Aperture Arrays.

The last example in the text results indirectly from the QUASAT times and the need to update the Westerbork array at about the same time. Through its compactness and cryogenic "simplicity" it is felt to be relevant to the design of the feed system for the SKA dishes.

Purposely, I did not address other more recent and significant VLBI developments e.g. related to real time data transport, networks and processing known to be relevant for the SKA as well.

\section{Ackowledgements}

In the text and through the references I tried to do justice to people who have been important to make the VLBI related instrumental developments at ASTRON in the early years possible while not forgetting the relevance to the SKA now. In all cases, Richard stands up as the initiator and critical customer in most cases deserving credits beyond being an impressive colleague, for instruments and astronomy but also as contributor to the structure and organization of it all!

\section{References}

[1] R.T. Schilizzi , G. K. Miley, A. van Ardenne, B. Baud, I. Baath, B.O. Rönnang, I.I.K. PaulinyToth, "High resolution observations of the compact central component in the giant radio source $3 \mathrm{C}$ 236”, Astr.\&Astroph., 77, pp 1-6, 1979

[2] J.P. Macquart, Transients, Aperture Arrays for the SKA/AAVP 2011 Workshop Dwingeloo 1216Dec. Dwingeloo, http://www.astron.nl/aavp2011/documents/Macquart_1PulsarsTransients13Dec2011.pdf.

[3] A. van Ardenne, “NFRA MKII VLBI System”, Internal Technical Rreport 168,1982

[4] A. B. J. Kokkeler, P. Fridman, A. van Ardenne, “ Degradation due to quantization noise in radio astronomy phased arrays”, Journal for Experimental Astronomy , Vol 11, 33 - 56, Feb. 2001

[5] A. van Ardenne, J. Buiter, R.Kiers, “Description of the Digital Combiner”, NFRA Note 362, Dec. 1981

[6] R.G. Strom and H.W. van Someren Greve, "Synchronous Aperture Synthesis Observations of Pulsars” Astroph. \& Space Sc., Vol 171, 351-355, 1990

[7] J.L.L. Voûte and P.C. van Haren, "PuManual; Manual for PUMA, the Pulsar Machine for the Westerbork Synthesis Array”, 1999 
[8] A. van Ardenne, J.D. Bregman, W. A. van Cappellen, G.W. Kant, J.G. bij de Vaate, “Extending the Field of View with Phased Array Techniques", Proc. IEEE, Special Issue on Radio Astronomy, vol.97, no8, pp 1531-1542, Aug. 2009

[9] R. Mailloux, “Electronically scanned arrays”, ISBN978-1598291827, 2007

[10] J. Hessels, O. Wucknitz, B, Stappers, “Time for Tune-up”, ASTRON-Jive Daily Image, 29 Oct, 2012

[11] A.E.E. Rogers, J.M. Moran, “Coherence limits for VLBI”, IEEE-IM, Vol IM-30, 4, 283-286, 1981

[12] A. van Ardenne, "Phase/Frequency noise of the Westerbork local oscillator system”, NFRA Note 463, Dec. 1984

[13] F. Biraud (Ed.), "Very Long Baseline Interferometric Techniques", Toulouse CNRS Workshop, Fr,, Aug-Sept 1982, Editions Cepadues, ISBN 2.85428.087.3, 1983

[14] H. Ames, S. Bolton, B.F. Burke, C. Christensen, V. Dhawan, B. Freland, D. and 43 more authors in alphabetical order, "Quasat: Technical aspects of the proposed mission”, ESA Spec.Publ., ESA SP-213, p27-99, 1984

[15] R.T. Schilizzi, “QUASAT-A space VLBI observatory”, Proc. Science workshopTorino, Italy, May 1987(A88-54076 24-90). Kluwer Acad. Publishers, pp 425-432, 1988

[16] J.L. Yen, K.I. Kellerman et al., "Real-time VLBI based on the use of the communications satellite”, Science, 289-291, 1977

[17] J. Bardin, S. Weinreb and D. Bagri, "Local Oscillator Distribution using a Geostationary Satellite”, Exp. Astronomy 2004 (Eds. P. Hall), 17, pp193-199, ISBN 1-4020-3798-8, Kluwer 2005

[18] D. Savini, G.Figlia, A. van Ardenne, K. v. Klooster, "A Triple Frequency Feed for the QUASAT Antenna”, IEEE Ant.\&Prop.Int.Symp., pp 342-345, Syracusa, 1988

[19] G.H. Tan,"Upgrade of the WSRT, The MultiFrequency Frontend”,Proc. $26^{\text {th }}$ EuMC, Vol 1, pp59-64, 1996 